\title{
COMMISSIONING OF THE COUPLED CYCLOTRON FACILITY AT NSCL *
}

\author{
P. Miller, F. Marti, D. Poe, M. Steiner, J. Stetson, X.Y. Wu, \\ NSCL, MSU, East Lansing, MI 48824, USA
}

\begin{abstract}
The Coupled Cyclotron Facility at Michigan State University is being completed with the experimental program scheduled to start in July 2001. First beam through the paired cyclotrons was obtained on 10 October 2000 .
\end{abstract}

\section{INTRODUCTION}

In the early 1990's the National Superconducting Cyclotron Laboratory (NSCL) at Michigan State University (MSU) explored different options for upgrading facilities to meet anticipated experimental demands for a wider variety and greater intensity of radioactive nuclear beams, which were being provided by the A1200 fragment separator.[1] One way to increase secondary beam intensity was to raise the primary beam energy, which achieves a large fraction of the maximum production rate at $400 \mathrm{MeV} / \mathrm{u}$. This was rejected on the basis of cost and timeliness for the intermediate term and two other options were explored and eventually implemented instead. These were: (1) to enhance transmission efficiency with a new higher acceptance particle separator, now also being commissioned as the A1900 [2], and (2) to increase primary beam intensities available from the K1200.

These considerations led in 1994 to the publication of a white paper [3] which included a refurbishment plan of the K500 cyclotron for use as an injector to the K1200. This scheme allowed higher intensities overall as well as raising energy limits for the heavier ions.

The US National Science Foundation approved this project in 1996. Construction started immediately, resulting in first beam through the coupled system in October, 2000.

\section{ECR AND INJECTION LINE}

NSCL presently operates two ECR's, one superconducting (SCECR) and the other (ARTEMIS [4]) with room temperature magnets built from a design based on the AECR at LBNL. Appropriate beam lines allow either source to inject beam into either cyclotron since stand-alone K1200 operation is still possible after the upgrade. Injected beam intensities are controlled by a series of attenuation screens in these beam lines allowing changes in factor-of-3 steps downwards from full beam to about $10^{-7}$ of that value. One problem addressed during reconstruction of the injection line into the K500 cyclotron was the effect of non-symmetric fringe fields.

Beam from the ECR sources is directed vertically down, horizontal, then vertically upwards along the K500 field axis (see Figure 1). K500 fringe field effects are already significant (300-400 G) at this upwards bend. In the previous configuration the bend was produced magnetically. The iron of this magnet and of the shielding on the horizontal beam line produced a significant asymmetry of the vertical field lines, disturbing the K500 solenoidal fringe field. To minimize this asymmetric situation we introduced dummy iron volumes that mimic the iron shielding around the beam line and replaced the 90 degree bending magnet with an electrostatic deflector

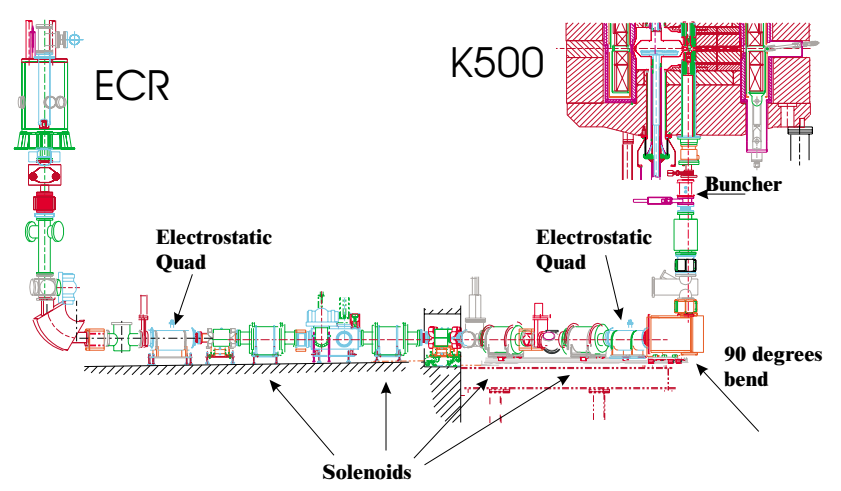

Figure 1: ECR to K500 low energy beam line.

enclosed in an iron box to reduce the effects of the fringe field. This configuration has provided a more symmetric fringe field thus simplifying beam tuning by reducing needed steering corrections.

\section{K500 CYCLOTRON}

The K500 cyclotron had to be completely disassembled and was rotated in order to direct the extracted beam to the K1200 instead of to the experimental vaults. Additionally, the magnetic circuit, cryostat and return yoke were modified for greater symmetry, the RF amplifiers were replaced, and the main magnet power supply was rebuilt. 
The K500 has shown a much-improved stability compared to its earlier operation. Figure 2 shows a probe trace from the K500 cyclotron. This probe consists of a differential wire followed by a main "block" and allows us to see the turn pattern at small radii as well as precession signature at larger radii. The K500 is operated in broad phase, multi-turn extraction mode, hence extraction efficiency is typically between 65 and $75 \%$. An interesting but not yet understood observation is shown in Figure 3 where K500 extracted current is plotted as a function of the buncher voltage.

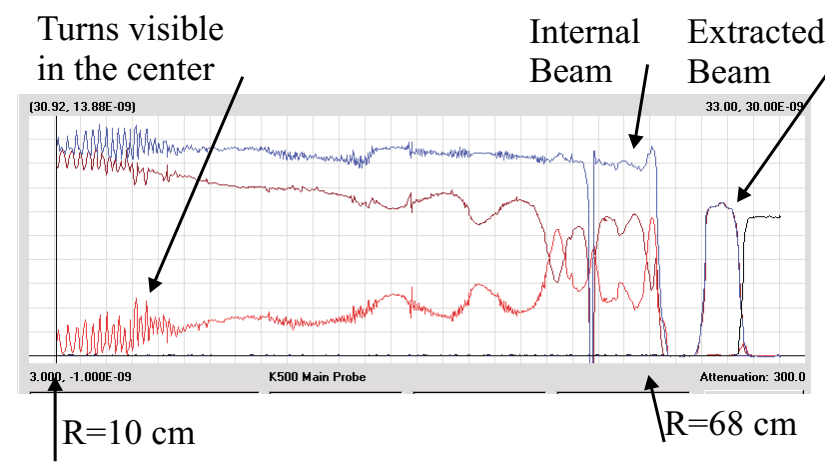

Figure 2: K500 beam probe trace.

By changing the attenuators we can change the beam intensity without modifying the injected phase space.

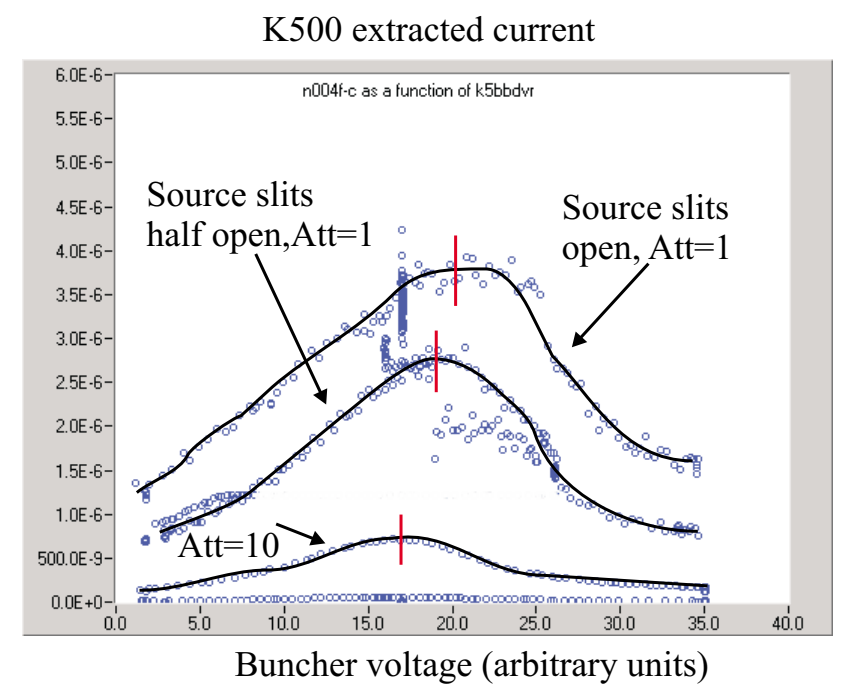

Figure 3: Extracted current from the K500 cyclotron as a function of the buncher voltage.

When plotting curves for various levels of attenuation they generally scale normally, obtaining the same bunching gain for all voltages. But when working at the highest intensities, with attenuation factors of 10 and 1 (no attenuation), behaviour is abnormal; the curves are not proportional to increasing current. The possibility of space charge effects had been explored but our models did not show any significant effects at these intensities.

\section{COUPLING LINE}

The coupling line transports the beam from the K500 to the K1200 with enough tuning flexibility to match the K500 extracted beam to the required phase space at the entrance of the K1200. For purpose of matching we have implemented an emittance and energy measurement system.

\section{K1200 CYCLOTRON}

\subsection{Injection Channel}

The injected beam path inside the K1200 cyclotron is shown in Figure 4. The dipole quadrupole combined magnet has a tapered pole shape and a single coil [5]. The quadrupole field is thus determined by the bending needed. Quadrupoles upstream are adjusted to match.

The label "Focusing bar" points to a passive element built in a similar way to our extraction focusing bars.

It is a conical cluster of three bars that produces a field gradient at the beam path. Beam also experiences transverse forces due to the RF field in the gap region. Consequently beam position at the stripper foil is phase dependent, and even initial tuning must be done with RF phases and voltage close to final values.

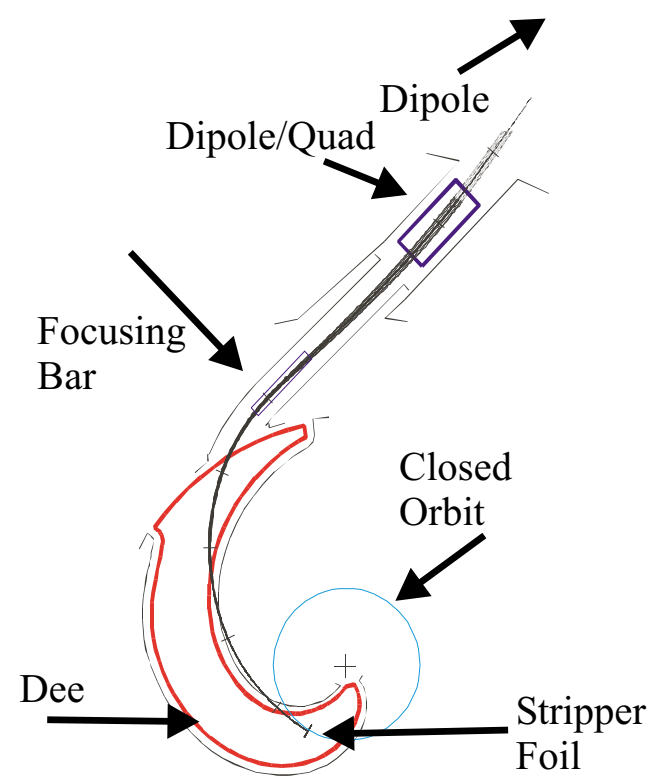

Figure 4: Injection channel path. A dipole is situated just outside the top of the figure. The thick red line indicates the dee profile.

\subsection{Stripper Foil Mechanism}

The stripper foil mechanism [6] places the foil at the matching point inside the dee with an accuracy of $0.3 \mathrm{~mm}$ and also holds a large reserve supply (31) of foils for immediate use.

A pair of hydraulic cylinders moves a platter in two directions to position the foil and another pair moves a 
bicycle chain to which the foils are attached. A ramp brings the foils from their horizontal, off the median plane position, to a vertical position, and later back to horizontal when no longer needed.

\subsection{Performance}

The first nuclear physics experiment was finished on June $4^{\text {th }}, 2001$, with the experimental program expected to start in July. The system performed smoothly with a beam attenuation factor of 30 and delivering $4.1 \times 10^{10}$ particles/second. We have not increased the intensity to the design values yet to avoid activating the components before a thorough check has been performed, along with a radiation safety system verification.

The K1200 extraction efficiency is in the $60-70 \%$ range. Overall efficiency for the whole system is currently approximately $2 \%$.

\section{A1900 FRAGMENT SEPARATOR}

The A1900 design put great emphasis on large acceptance to accommodate high-emittance secondary beams and has a solid angle of $8.0 \mathrm{msr}$, as compared with

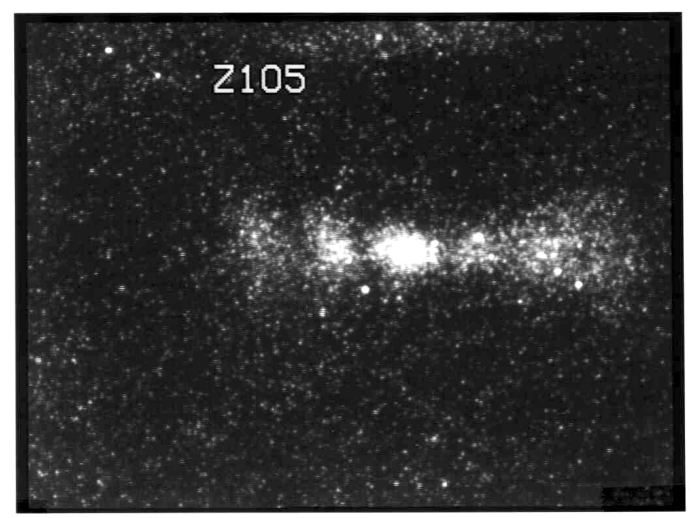

Figure 5: Picture of a beam of fragments from ${ }^{40} \mathrm{Ar}$ impinging on a scintillator viewer at the A1900 focal plane, taken with an image-intensified camera. The bright spot in the center is approximately $5 \mathrm{~mm}$ wide by $3.5 \mathrm{~mm}$ tall.

$0.8 \mathrm{msr}$ for the A1200. The separation of individual isotopes requires small beam spots, and 16 sextupole magnets and an equal number of octupoles were incorporated into the design of the superconducting beamline to achieve high resolution. Early experiments show that these correction magnets are indeed important to meet the transmission and resolution requirements.

An intermediate energy degrader was placed at the central image of the A1900, and the magnetic rigidity of the second half of the device was lowered to direct some of the fragments onto the focal plane scintillator viewer (see Figure 5).

As different isotopes lose different amounts of energy in the degrader, they become spatially separated and can be selected with slits for transport to the experimental vaults. An advantage of using a fragment beam rather than primary beam for optics development is the large angular and momentum spread of the fragments, which allows the experimenter to probe a large part of the A1900 acceptance. The momentum spread of these beams extends over the device's momentum acceptance of $\pm 2.5 \%$.

Figure 6 shows two spectra taken on-line with particle detectors at the A1900 focal plane. The left panel shows the particle identification spectrum, with the energy-loss signal of a PIN diode on the ordinate, and the time-offlight on the abscissa. The nucleus of interest, ${ }^{33} \mathrm{Al}$, has been circled. The right panel has the same ordinate, but the abscissa is the horizontal beam position as recorded in a tracking detector, with particles spanning a total width of about $5 \mathrm{~cm}$. The picture shows how slits cutting into the beam from both sides can be used to produce a relatively "clean" beam of the desired isotope, and illustrates that the A1900 is working well at this stage of its development.

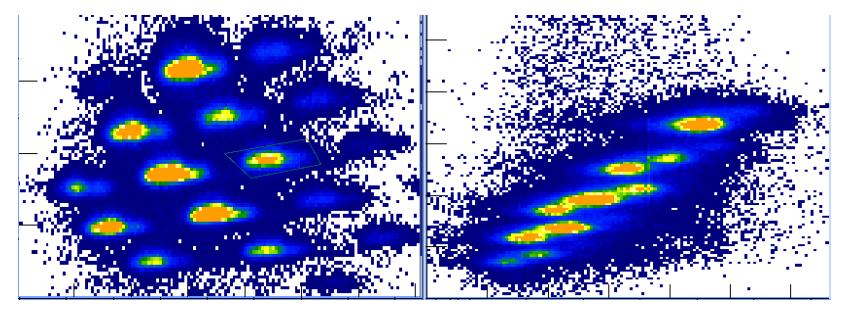

Figure 6: Spectra taken with the A1900 particle detection system. Spectrometer settings and isotopes are not the same as in the previous picture. See text for description.

\section{REFERENCES}

[1] B.M. Sherrill, D.J. Morrissey, J.A. Nolen Jr. and J.A. Winger, "The A1200 Projectile Fragment Separator", Nucl. Inst. \& Meth. in Physics Research B56/57 (1991) 1106-1110.

[2] D.J. Morrissey, NSCL staff, "A New High-Resolution Separator for High-Intensity Secondary Beams", Nucl. Inst. \& Meth. in Physics Research B126 (1997) 316-319.

[3] The K500-K1200, A Coupled Cyclotron Facility at the NSCL, MSU, MSUCL-939, July 1994.

[4] H. Koivisto, et al., To be published in Proc. Of the Workshop on the Production of Intense Beams of Highly Charged Ions, Catania, Italy, September 24-27, 2000, Conference Proceeding of Societa Italiana de Fisica.

[5] D. Lawton, F. Marti, X. Y. Wu, A. F. Zeller, "Design of the Injection Channel Magnets for the K1200 Superconducting Cyclotron", to be published in Proceedings of Cyclotrons 2001 Conference, May 2001, E. Lansing, MI www.nscl.msu.edu/cyc2001.

[6] J. Bonofiglio, S. Alfredson, F. Marti, A. Rodriguez, G. Stork, "Stripper Foil Mechanism for the K1200 Cyclotron" to be published in Proceedings of Cyclotrons 2001 Conference, May 2001, E. Lansing, MI www.nscl.msu.edu/cyc2001.

* Work supported by NSF Grant No. PHY-9528844 